\title{
Efficacy and Moderators of Internet-Based Interventions in Adults with Subthreshold Depression: An Individual Participant Data Meta-Analysis of Randomized Controlled Trials
}

\author{
Jo Annika Reins ${ }^{a} \quad$ Claudia Buntrock $^{b}$ Johannes Zimmermann ${ }^{c}$ Simon Grund ${ }^{d}$ \\ Mathias Harrer ${ }^{\text {b }}$ Dirk Lehr ${ }^{\text {a }}$ Harald Baumeister $^{\mathrm{e}}$ Kiona Weisel $^{\mathrm{b}}$ \\ Matthias Domhardt ${ }^{\mathrm{e}}$ Kotaro Imamura $^{\mathrm{f}}$ Norito Kawakami $^{\mathrm{f}}$ Viola Spek $^{\mathrm{g}}$ \\ Stephanie Nobis $^{\mathrm{h}}$ Frank Snoek $^{\mathrm{i}}$ Pim Cuijpers $^{j}$ Jan Philipp Kleink ${ }^{\mathrm{k}}$ \\ Steffen Moritz' David Daniel Ebert ${ }^{\mathrm{i}}$

\begin{abstract}
a Department of Health Psychology and Applied Biological Psychology, Institute of Psychology, Leuphana University Lueneburg, Lueneburg, Germany; ${ }^{b}$ Department of Clinical Psychology and Psychotherapy, Friedrich-Alexander University Erlangen-Nuremberg, Erlangen, Germany; ${ }^{C}$ Department of Psychology, University of Kassel, Kassel, Germany; ${ }^{d}$ Leibniz Institute for Science and Mathematics Education, University of Kiel, Kiel, Germany; ${ }^{e}$ Department

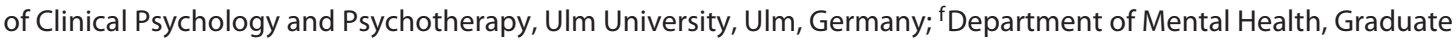
School of Medicine, University of Tokyo, Tokyo, Japan; ${ }^{9}$ Fontys University of Applied Sciences, Eindhoven,

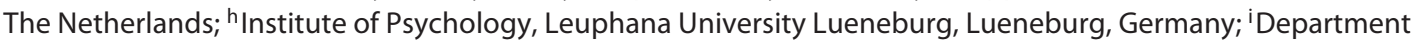
of Medical Psychology, Amsterdam University Medical Centers, Vrije Universiteit Amsterdam, Amsterdam, The Netherlands; 'Department of Clinical, Neuro- and Developmental Psychology, Amsterdam Public Health Research Institute, Vrije Universiteit Amsterdam, Amsterdam, The Netherlands; ${ }^{k}$ Department of Psychiatry and Psychotherapy, Luebeck University, Luebeck, Germany; 'Department of Psychiatry and Psychotherapy, University Medical Center Hamburg-Eppendorf, Hamburg, Germany
\end{abstract}

\section{Keywords}

Individual participant data meta-analysis - Moderators . eHealth · Online therapy · Subclinical depression · Minor depression

\section{Abstract \\ Introduction: Evidence on effects of Internet-based inter- ventions to treat subthreshold depression (sD) and prevent the onset of major depression (MDD) is inconsistent. Objec-}

tive: We conducted an individual participant data metaanalysis to determine differences between intervention and control groups (IG, CG) in depressive symptom severity (DSS), treatment response, close to symptom-free status, symptom deterioration and MDD onset as well as moderators of intervention outcomes. Methods: Randomized con-

Trial registration: PROSPERO database, registration No. CRD42017058585.
(C) 2020 The Author(s)

Published by S. Karger AG, Basel

This article is licensed under the Creative Commons AttributionNonCommercial-NoDerivatives 4.0 International License (CC BYNC-ND) (http://www.karger.com/Services/OpenAccessLicense) Usage and distribution for commercial purposes as well as any distribution of modified material requires written permission.
David Daniel Ebert

Department of Medical Psychology, Vrije Universiteit Amsterdam De Boelelaan 1105

NL-1081 HV Amsterdam (The Netherlands)

d.d.ebert@vu.nl 
trolled trials were identified through systematic searches via PubMed, PsycINFO, Embase and Cochrane Library. Multilevel regression analyses were used to examine efficacy and moderators. Results: Seven trials (2,186 participants) were included. The IG was superior in DSS at all measurement points (posttreatment: 6-12 weeks; Hedges' $\mathrm{g}=0.39$ [95\% Cl: 0.25-0.53]; follow-up 1: 3-6 months; $\mathrm{g}=0.30[95 \% \mathrm{Cl}$ : $0.15-0.45$ ]; follow-up 2: 12 months, $g=0.27$ [95\% Cl: $0.07-$ $0.47]$, compared with the CG. Significantly more participants in the IG than in the CG reached response and close to symptom-free status at all measurement points. A significant difference in symptom deterioration between the groups was found at the posttreatment assessment and follow-up 2. Incidence rates for MDD onset within 12 months were lower in the IG (19\%) than in the CG (26\%). Higher initial DSS and older age were identified as moderators of intervention effect on DSS. Conclusions: Our findings provide evidence for Internet-based interventions to be a suitable low-threshold intervention to treat individuals with $\mathrm{SD}$ and to reduce the incidence of MDD. This might be particularly true for older people with a substantial symptom burden.

(C) 2020 The Author(s)

Published by S. Karger AG, Basel

\section{Introduction}

Subthreshold depression (sD) is highly prevalent [1], associated with poorer quality of life [2], a higher risk of developing major depression (MDD) $[3,4]$, increased mortality [5] and increased use of health care services [6], as well as immense economic costs [7].

Although a meta-analysis on Internet-based interventions to treat $\mathrm{sD}$ found small-to-moderate effects for psychological interventions on depressive symptom severity (DSS) after treatment compared to treatment as usual [8], uptake rates of preventive interventions are low [9]. Internet-based interventions might overcome some of the barriers associated with traditional mental health care services, e.g., living in underserved areas, and fear of negative social consequences $[10,11]$. Meta-analyses have shown positive effects of Internet-based interventions for depression [12, 13], with the National Institute for Health and Care Excellence guidelines recommending such interventions as a treatment option in the management of subthreshold persistent depressive symptoms and mild to moderate depression [14].

Several studies have investigated the efficacy of Internet-based interventions for the treatment of $\mathrm{sD}$ and the prevention of MDD. Three meta-analyses summarizing these studies found significant improvements in DSS af- ter treatment with small to moderate effect sizes (standardized mean difference $[S M D]=0.25,95 \%$ confidence interval, CI: 0.09-0.41 [15]; SMD $=0.28,95 \%$ CI: 0.140.42 [16]; SMD $=0.35,95 \%$ CI: 0.12-0.57 [17]). However, statistical comparisons based on group means provide limited information about clinical significance on an individual level, such as treatment response and symptomfree status [18]. In addition, evidence on the long-term efficacy remains inconsistent, with some randomized controlled trials (RCTs) showing a superiority of Internet-based programs over control conditions at follow-up [19-21], while others did not [22-24]. With regard to the prevention of MDD onset, evidence is scarce but promising with hazard ratios ranging from 0.22 (95\% CI: $0.06-$ 0.75 [25]) to 0.59 (95\% CI: 0.42-0.82 [21]). Moreover, research on negative outcomes, such as symptom deterioration, in the treatment of $\mathrm{sD}$ is rare but of high clinical relevance [26].

However, due to insufficient power in single RCTs [27], there is little evidence on whether all subgroups of participants with $\mathrm{sD}$ benefit from Internet-based interventions. Moderator analyses could identify different outcome patterns between participants and provide a basis for choosing the best fitting intervention for a given individual [28]. By pooling raw data of individual trials (e.g., individual participant data meta-analysis, IPD-MA), it is feasible to perform analyses not reported in original studies and to obtain sample sizes with sufficient power to investigate effects in relevant subgroups and explore intervention and participants' characteristics as moderators of intervention outcome [29].

The aim of the present study was thus to investigate the short- and long-term effects of Internet-based interventions in comparison to control groups like treatment as usual or waiting list groups in adults with sD on DSS, treatment response, close to symptom-free status, symptom deterioration and depression onset as well as moderators on the individual and study level, using an IPD-MA approach.

\section{Materials and Methods}

\section{Registration and Study Protocol}

This IPD-MA was registered in the PROSPERO register (CRD42017058585). It was conducted and reported according to methodological guidelines for IPD-MA [30, 31]. Further details of the study can be found in the published study protocol about the planned IPD-MA on interventions for the treatment of $\mathrm{sD}$ and prevention of MDD [32]. Deviations from the protocol can be found in online supplement 1 (for all online suppl. material, see www.karger.com/doi/10.1159/000507819). 
Eligibility Criteria/Identification and Selection of Studies

We included RCTs in which effects of an Internet-based psychological intervention were compared with a comparison group in adults with no MDD at baseline according to DSM/ICD criteria (assessed with a standardized diagnostic categorical assessment, either via interview or self-administered questionnaire) and scoring above a cut-off on a self-rating depression questionnaire or meeting criteria for minor depression according to the Diagnostic and Statistical Manual of Mental Disorders (DSM). RCTs with mixed sD/MDD samples were included and MDD cases at baseline were removed from the data set. Studies were excluded if they were not published in English, German, Spanish or Dutch.

To identify potential studies, we used a database of articles on the psychological treatment of depression, which is described in detail elsewhere [33]. This database contains studies that have been identified using PubMed, PsycINFO, Embase and the Cochrane Central Register of Controlled Trials. In addition, previous metaanalyses on depression prevention and treatment of $\mathrm{sD}[16,17]$ were reviewed, and renowned authors in the field of depression prevention were asked if they were aware of any other relevant study to ensure that no RCT was overseen. Studies published until May 30, 2017, were considered for inclusion.

\section{Data Collection, Extraction and Preparation}

Authors of eligible articles were contacted for permission to use their data sets. Reminders were sent after 2 weeks and if necessary after 1 month. In case of non-response, we excluded the trial. Authors were asked to provide data on sociodemographic, clinical and intervention-related characteristics. Potential moderators on participant level were identified by exploring variables that have been found to predict long-term outcome in depression [34, 35]. Next, moderators were extracted from studies according to the amount of available/missing data and the bivariate associations with outcome measures in the intervention and control group. Detailed information can be found in online supplement 2. Finally, we combined all individual data sets into a merged data set, using a generic standardized protocol for integrating IPD sets [36]. All postintervention assessments were pooled and treated as one assessment. Follow-ups were categorized into appropriate categories (FU1: 3-6 months, FU2: 12 months).

\section{Risk of Bias Assessment}

The validity of the included studies was assessed using four criteria from the Cochrane Risk of Bias assessment tool [37] including adequate generation of allocation sequence, allocation concealment, blinding of assessors and dealing with incomplete outcome data (e.g., intention-to-treat analyses were assessed as positive). For this assessment only the information which was actually reported in the papers was used, to ensure a consistent procedure across studies and to reduce the risk of bias based on what was reported and what was not. The quality assessment was carried out independently by two researchers (C.B., J.R.). Disagreements were resolved by discussion.

\section{Missing Data}

The IPD-MA was conducted according to the intention-totreat principle. Missing data were handled using a fully conditional specification approach to multiple imputation using the $\mathrm{R}$ package mice [38]. The imputation procedure was set up in such a way that it would incorporate the nested structure of the data with in- dividual participant data nested within studies, include all available individual and study level characteristics as predictors of missing data and allow for heterogeneity in treatment effects across studies as well as treatment-by-moderator interactions [3942]. The nested structure of the data was modelled using dummy indicators for each study $[40,43]$. This was in contrast to the registered protocol but required because methods based on mixedeffects models showed significant problems with model convergence, making it unfeasible to use them for the imputation of missing data [44]. The imputations were carried out separately for each treatment group (intervention vs. control), thus taking both heterogeneity and possible treatment-by-moderator interactions into account. Both sporadically and systematically missing values were imputed $[41,42]$. All variables at the study and participant level were considered as predictors of missing data. However, due to systematic missing data in some studies and multicollinearity between study-level predictors (e.g., between study ID and intervention format), we encountered estimation problems with the effects of some study-level predictors. In such a case, the procedure was adjusted such that it included the largest subset of predictors for which estimation was possible. Based on this procedure, we generated 100 imputed data sets, each after 50 iterations of the imputation algorithm. A detailed documentation of the procedure can be requested from the corresponding author.

\section{Outcome Measures}

The following types of outcome criteria were used: (a) DSS, (b) treatment response (evaluated in two different ways: (1) reliable change index [RCI] [18]; (2) 50\% reduction in symptoms), (c) close to symptom-free status (e.g., scoring below a predefined cut-off score, i.e. 13.00 for BDI-II [45, 46] and 11.88 for CES-D [47]), (d) symptom deterioration (defined by (1) RCI; (2) 50\% increase in symptoms) and (e) time to MDD onset (e.g., DSM-IV criteria assessed with the telephone-administered SCID or the web version of the WHO Composite International Diagnostic Interview, CIDI). Standardized depression outcome measures (BDI-II [48]; CES-D [49]) were transformed into standardized $t$ value scores (i.e., population mean of 50 and standard deviation of 10), using the common metric approach [50]. After the imputation procedure the common metric was backtransformed into the original metric and then transformed into treatment response, close to symptom-free status and deterioration rates.

\section{Moderators of Intervention Effects on DSS}

The following sociodemographic characteristics were included: sex [51-54], age [51, 55], relationship status $[51,56]$, education $[57$, 58] and employment [56]. Initial DSS [59-62], initial anxiety symptoms [63] measured with the HADS-A questionnaire or via CIDI interview, comorbid mental health problems [55] measured with distress scales (K6, PAID) or the Web Screening Questionnaire, previous psychotherapy for depression, use of antidepressants [58] and chronic medical conditions $[51,55,59]$ were examined in terms of clinical characteristics. The format of the Internet intervention (e.g., guided, unguided) as well as the length of the treatment [64] were analysed as intervention characteristics on study level.

IPD Meta-Analysis

For the IPD-MA we utilized a one-step data analysis approach because the effects of moderators and study level covariates can be examined more precisely, compared to the two-step procedure 
Table 1. Selected characteristics of eligible randomized controlled trials examining the effects of psychological treatments of subthreshold depression in adults

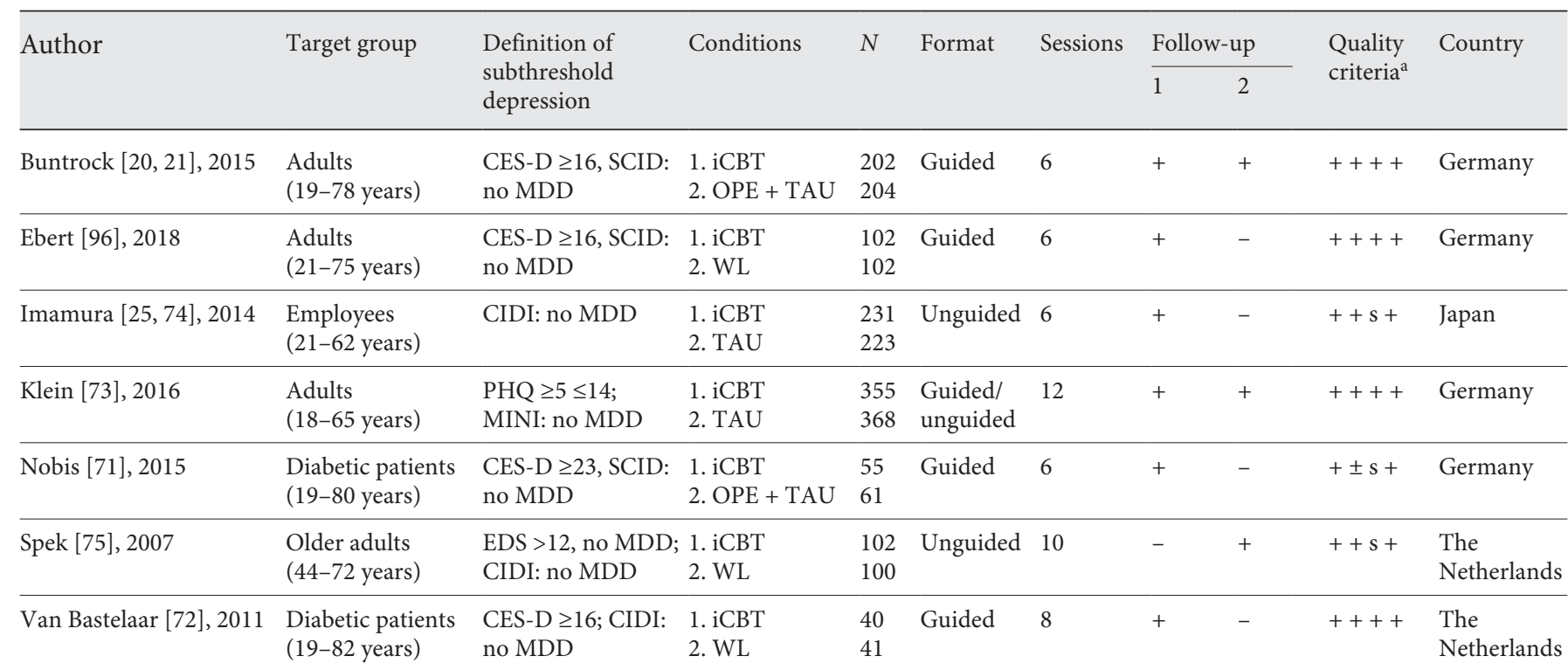

CES-D, Center for Epidemiological Studies Depression Scale; SCID, Semi-Structured Clinical Interview for DSM-4 or -5; CIDI, Composite International Diagnostic Interview; PHQ, Patient Health Questionnaire; MINI, Mini International Neuropsychiatric Interview; EDS, Edinburgh Depression Scale; MDD, major depressive disorder; iCBT, internet-based cognitive-behavioural therapy; OPE, online psychoeducation; TAU, treatment as usual; WL, waiting list. ${ }^{\text {a }}$ In this column a positive $(+)$, negative $(-)$ or unclear $( \pm)$ sign is given for four quality criteria, respectively: allocation sequence; concealment of allocation to conditions; blinding of assessors; intention-to-treat analyses; all studies were rated as fulfilling the "intention-to-treat" criteria, as multiple imputation was used for all studies to handle missing data. An "s" indicates that only self-report instruments were used as outcome measure.

[65]. In the protocol [32], we defined that we additionally planned to conduct a two-step meta-analysis on study level to examine whether studies that did not provide data might bias the results of our IPD-MA. However, this was not necessary, as we were able to include the primary data of all identified trials on Internet-based interventions to treat sD in the current study. All analyses except for the survival analyses were carried out with the imputed data set. Due to the relatively high missing rates of FU1 and FU2, additional sensitivity analyses were performed and reported with the original data for our primary outcome (DSS).

\section{Effects on DSS}

We used a multilevel regression analysis predicting DSS scores from treatment group while controlling for baseline DSS. We included both a random intercept and random slope for the treatment effects to capture both unobserved heterogeneity between study populations (intercept) and study efficacy (slope). Models were fitted to all multiply imputed data sets, and final parameter estimates were aggregated via Rubin's rule [66], using the lme4 [67] and mitml [68] packages in $\mathrm{R}$ version 3.5.2. Hedges' $\mathrm{g}$ and corresponding 95\% CI were calculated as a standardized effect size measure, using the populations' standard deviation $(\mathrm{SD}=10)$ of the common metrics.

Effects on Response, Close to Symptom-Free Status and Deterioration

We used multilevel logistic regression analyses including both a random intercept and random slope for the treatment effects. We proceeded to calculate odds ratios (OR) and 95\% CI to further investigate differences between intervention groups. In addition, we calculated the numbers needed to benefit (NNTB) or the numbers needed to harm (NNTH) in order to achieve one additional response, respectively, close to symptom-free status or case of deterioration as compared to the control group [69].

\section{Effects on Depression Onset}

Time to MDD onset was expressed in weeks. The mean survival time was calculated as the area under the Kaplan-Meier survivor function within the 12-month study period. Differences in survivor functions between intervention groups were analysed using the log rank test. The nested structure of the data was not taken into consideration for these two analyses. In addition, we used Cox proportional hazard regression models (based on mixed-effects models to handle the nested data structure) controlling for initial DSS to investigate differences in time to onset of MDD between intervention and control groups. The proportional hazards assumption was evaluated using the scaled Schoenfeld residual test [70].

\section{Moderators of Intervention Effects on DSS}

We explored moderators of the intervention effect by including selected participant level and study level variables as well as their interaction with the intervention in the multilevel regression analyses. To reduce the complexity for moderator analyses we focused on DSS at posttreatment assessment only. 
Table 2. Means, standard deviations and percentage of outcome variables at baseline, posttreatment time point (post), follow-up 1 and follow-up 2

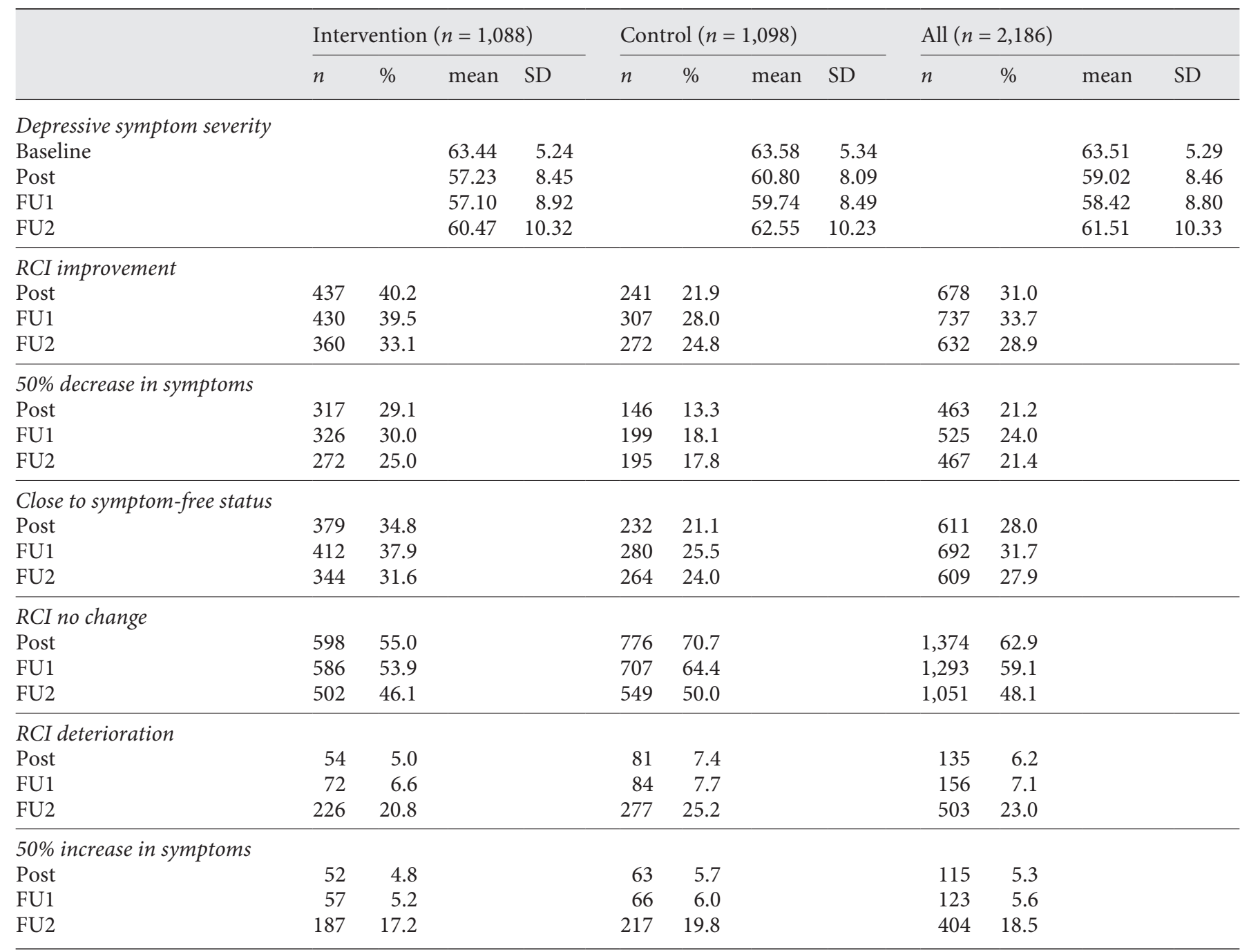

Percentages and absolute numbers are estimated based on multiple imputation. $N$, number of participants; SD, standard deviation; FU, follow-up; RCI, reliable change index.

\section{Results}

\section{Study Selection and IPD Obtained}

The flow chart inclusion of studies (online supplement 3 ) shows the selection process for included studies. The systematic search on psychological treatments for subthreshold depression resulted in a total of 16,407 abstracts (12,196 after the removal of duplicates), of which 1,885 full-text articles of RCTs on treatments for depression were retrieved. Of the 27 identified studies investigating the efficacy of psychological treatments for $\mathrm{sD}$, we were able to obtain raw data from 21 data sets. While preparing the pooled data set, 5 further studies were published and could be integrated, resulting in a final sample of 26 primary study data sets. Of those 26 studies, 7 evaluated an Internet-based intervention and were thus included in the current IPD-MA.

\section{Study Characteristics}

Primary data had been successfully obtained from all of the 7 identified studies. An overview of study characteristics can be found in Table 1. Three of these studies included participants diagnosed with MDD [71-73], and one study additionally included participants with no ele-
98

Psychother Psychosom 2021;90:94-106 DOI: $10.1159 / 000507819$
Reins et al. 
Table 3. Multilevel regression analyses predicting depressive symptom severity scores from treatment group

\begin{tabular}{|c|c|c|c|c|c|c|c|c|c|}
\hline \multirow[t]{2}{*}{ DSS } & \multicolumn{3}{|c|}{ Posttreatment time point } & \multicolumn{3}{|c|}{ Follow-up 1} & \multicolumn{3}{|c|}{ Follow-up 2} \\
\hline & est. & SE & $p$ & est. & $\mathrm{SE}$ & $p$ & est. & SE & $p$ \\
\hline \multicolumn{10}{|l|}{ Fixed effects } \\
\hline Intercept $\left(b_{0}\right)$ & 17.268 & 2.352 & $<0.001$ & 14.945 & 2.618 & $<0.001$ & 24.743 & 3.544 & $<0.001$ \\
\hline Condition $\left(b_{1}\right)$ & -3.874 & 0.705 & $<0.001$ & -3.028 & 0.767 & $<0.001$ & -2.688 & 1.037 & 0.01 \\
\hline Baseline DSS $\left(b_{2}\right)$ & 0.685 & 0.035 & $<0.001$ & 0.705 & 0.039 & $<0.001$ & 0.558 & 0.047 & $<0.001$ \\
\hline \multicolumn{10}{|l|}{ Random effects (var) } \\
\hline Intercept $\left(\tau_{0}^{2}\right)$ & 2.559 & & & 3.247 & & & 24.61 & & \\
\hline Condition $\left(\tau^{2}{ }_{1}\right)$ & 2.249 & & & 2.437 & & & 3.384 & & \\
\hline $\begin{array}{l}\text { Covariance intercept- } \\
\text { condition }\left(\tau 2^{0}{ }_{1}\right)\end{array}$ & -1.101 & & & -1.231 & & & 4.136 & & \\
\hline
\end{tabular}

The multilevel regression analyses were controlled for initial depressive symptom severity. $b_{0}$, Y-intercept; $b_{1}$, treatment condition; $b_{2}$, initial depressive symptom severity; $\tau^{2}{ }_{0}$, intercept variance; $\tau^{2}$, slope variance; est., estimate; SE, standard error; $\mathrm{p}$, probability.

vated depressive symptoms [74]. These participants were excluded on an individual basis using the primary data. As one RCT was a three-arm design [75] we only included the condition of interest (online treatment) compared to the control condition and neglected the third arm (face-toface treatment). This process resulted in a data set with the primary data from 2,186 cases $(1,088$ in the intervention groups, IGs, and 1,098 in the control groups, CGs).

\section{Risk of Bias Assessment}

The quality assessment of the included studies based on the published reports can be found in Table 1. Overall risk of bias was low. All studies reported an adequate sequence generation and reported blinding of outcome assessors or used self-report outcomes. Six of 7 studies reported an allocation to conditions by an independent (third) party; in 1 study there was no information given. All studies followed an intention-to-treat principle. Six of 7 studies met all 4 quality criteria; the remaining study met 3 of 4 criteria. Agreement between raters on the risk of bias was high across studies with Cohen's $\kappa=0.94$.

\section{Demographics and Clinical Characteristics}

Participant characteristics at baseline are shown in online supplement 4 . More than half of the participants were female $(58.6 \%)$, with an average age of 44 years $(\mathrm{SD}=11.6)$, in a relationship (58.2\%) and employed (78.6\%). At baseline, $25.8 \%$ of the participants used antidepressants. Moreover, $45.2 \%$ of the individuals had already undergone psychotherapy at some point in their lives, $33.5 \%$ suffered from comorbidities, and $51.4 \%$ reported chronic medical condi-

IPD-MA on Internet-Based Interventions in Adults with Subthreshold Depression tions. The mean initial depressive symptom level (common metrics) was $63.51(\mathrm{SD}=5.29)$, which equals a value of 1.3 SD above the population average. There were no clinically important differences between treatment conditions in terms of any baseline characteristics. The most common patterns of missing values can be found in online supplement 5. More than half of the participants (58.6\%) did not provide data on chronic medical conditions. There was also a remarkable amount of missing data in terms of DSS at FU2 (58.0\%; see also Table 1 for an overview which trials assessed data at FU1 and at FU2), previous psychotherapy (56.7\%) and comorbid anxiety (49.5\%).

\section{Effects on DSS}

Means, standard deviations and percentage of multiply imputed outcome variables at baseline, posttreatment, FU1 and FU2 are displayed in Table 2. Effects on short- and long-term symptom severity are displayed in Table 3. A statistically significant difference in DSS was found between the IG and the CG at posttreatment assessment $(\mathrm{b}=-3.874,95 \% \mathrm{CI}:-5.26,-2.49 ; t(17,229)=$ $-5.49, p<0.001$; Hedges' $g=0.39$ [95\% CI: $0.25-0.53]$ ), at FU1 $(b=-3.028,95 \%$ CI: $-4.53,-1.52 ; t(4,467)=-3.95$, $p<0.001 ; \mathrm{g}=0.30$ [95\% CI: 0.15-0.45]) and at FU2 (b = $-2.688,95 \%$ CI: $-4.72,-0.66$; $t(549)=-2.59, p=0.010$; $\mathrm{g}=0.27$ [95\% CI: 0.07-0.47]). Slope variance was relatively low at posttest time point compared to FU1 and FU2 (Table 3). This rise in slope variance may be interpreted as an increase in the heterogeneity of intervention effects over time. Therefore, we repeated the analyses for FU1 and FU2 with the studies that provided original data 
Table 4. Results from separate multilevel regression analyses on the effects of putative moderators on differential change in depression severity from baseline to posttreatment assessment

\begin{tabular}{lllllll}
\hline \multirow{2}{*}{ Baseline variable } & \multicolumn{5}{l}{ Interaction: baseline variable $\times$ treatment condition } \\
\cline { 2 - 7 } & estimate & $\mathrm{SE}$ & \multicolumn{1}{l}{$t$ value } & $p(<|\mathrm{t}|)$ & $\tau^{2}{ }_{0}$ & $\tau^{2}{ }_{1}$ \\
\hline Age & -0.066 & 0.033 & -2.002 & 0.045 & 2.812 & 1.972 \\
Gender & -0.799 & 0.784 & -1.02 & 0.308 & 2.568 & 1.998 \\
Relationship & -0.549 & 0.707 & -0.776 & 0.438 & 2.506 & 2.281 \\
Employment & 0.105 & 0.903 & 0.116 & 0.908 & 2.612 & 2.256 \\
Previous psychotherapy & 0.816 & 0.960 & 0.849 & 0.396 & 2.671 & 2.365 \\
Depression medication & 0.594 & 0.090 & 0.600 & 0.548 & 2.671 & 2.312 \\
Comorbidities & -0.816 & 0.846 & -0.965 & 0.335 & 2.825 & 1.904 \\
Chronic medical conditions & -0.306 & 1.392 & -0.22 & 0.826 & 2.639 & 2.197 \\
Comorbid anxiety & -0.950 & 0.905 & -1.049 & 0.295 & 2.996 & 2.086 \\
Initial symptom severity & -0.182 & 0.069 & -2.641 & 0.008 & 2.197 & 1.391 \\
Format & -1.797 & 0.944 & -1.905 & 0.057 & 2.655 & 0.786 \\
Number of sessions & -0.001 & 0.256 & -0.007 & 0.994 & 2.580 & 2.443 \\
Education & -0.566 & 0.605 & -0.936 & 0.350 & 2.597 & 2.621 \\
\hline
\end{tabular}

All analyses were controlled for initial depressive symptom severity. SE, standard error; $\tau^{2}{ }_{0}$, intercept variance; $\tau^{2}{ }_{1}$, slope variance; gender $(0=$ male, $1=$ female, $2=$ other $)$; relationship $(0=\operatorname{single} /$ divorced $/$ separated/widowed, $1=$ married/in a relationship); employment $(0=$ no, $1=$ yes $)$; previous psychotherapy $(0=$ no, $1=$ yes $)$; depression medication $(0=$ no, $1=$ yes $)$, comorbidities $(0=$ no, $1=$ yes $)$, chronic medical conditions $(0=$ no, $1=y e s)$, comorbid anxiety $(0=$ no, $1=$ yes $)$, format $(0=$ unguided, $1=$ guided $)$; education $(0=$ no $=0-5$ years, $1=$ low $=$ $6-9$ years, $2=$ middle $=10-12$ years, $3=$ high $=13-17$ years, $4=$ very high $=18+$ years $)$.

for these measurement points $(n=1,329 ; 61 \%$ of the total sample; online supplement 6 ). The results remained essentially the same but the group difference at FU2 became scarcely not significant $(p=0.055)$.

\section{Effects on Response, Close to Symptom-Free Status and Deterioration \\ Response}

Rates of response, close to symptom-free status and deterioration are displayed in Table 2 . Significantly more participants in the IG showed a reliable improvement, both based on the RCI and 50\% reduction in DSS compared to controls (RCI: OR $=2.46$; 95\% CI: $1.99-3.04 ; p<$ $0.001 ; 50 \%$ reduction: $\mathrm{OR}=2.72 ; 95 \%$ CI: $2.12-3.49 ; p<$ $0.001)$. Differences remained statistically significant at FU1 (RCI: OR $=1.73 ; 95 \%$ CI: $1.40-2.15 ; p<0.001 ; 50 \%$ reduction: $\mathrm{OR}=1.96 ; 95 \% \mathrm{CI}: 1.54-2.49 ; p=0.001)$, and at FU2 (RCI: $\mathrm{OR}=1.66 ; 95 \% \mathrm{CI}: 1.18-2.34 ; p=0.004 ; 50 \%$ reduction: $\mathrm{OR}=1.64 ; 95 \% \mathrm{CI}: 1.11-2.41 ; p=0.013)$. The NNTBs based on the RCI ( $50 \%$ symptom reduction) were 5.49 (95\% CI: 4.54-6.94) [NNTB = 6.31 (95\% CI: 5.218.02)] at posttreatment assessment, 8.65 (95\% CI: 6.4513.11) $[\mathrm{NNTB}=8.45$ (95\% CI: $6.50-12.06)]$ at FU1 and 12.03 (95\% CI: 8.26-22.08) $[\mathrm{NNTB}=13.81$ (95\% CI: 9.38-26.21)] at FU2.
Close to Symptom-Free Status

At the posttreatment time point, at FU1 and at FU2 significantly more participants of the IG reached a close to symptom-free status, compared to controls (post: OR $=2.12$; 95\% CI: $1.69-2.65, p<0.001 ; \mathrm{FU} 1: \mathrm{OR}=1.82 ; 95 \%$ CI: $1.46-2.26$; $p<0.001$; FU2: OR $=1.61 ; 95 \%$ CI: $1.09-$ 2.38 ; $p=0.017$ ). The NNTBs were 7.30 (95\% CI: $5.74-$ 10.02 ) at posttreatment assessment, 8.09 (95\% CI: $6.16-$ 11.77) at FU1 and 13.20 (95\% CI: 8.83-26.12) at FU2.

\section{Deterioration}

At the posttreatment assessment, the risk of deterioration, both based on the RCI and 50\% increase in DSS, was reduced in the IG compared to the CG but the difference was statistically significant only in the RCI at the postmeasurement time point $(\mathrm{OR}=0.65$; 95\% CI: $0.43-0.98$; $p=0.038)$ and at FU2 (OR $=0.70 ; 95 \%$ CI: $0.50-0.99 ; p=$ $0.046)$, but not at FU1 (OR $=0.85 ; 95 \%$ CI: $0.58-1.25 ; p=$ 0.412 ). There was no statistically significant difference in $50 \%$ increase at any measurement point (post: $\mathrm{OR}=0.82$; 95\% CI: 0.53-1.25; $p=0.345$; FU1: OR $=0.84$; 95\% CI: $0.55-1.29 ; p=0.419 ; \mathrm{FU} 2: \mathrm{OR}=0.82 ; 95 \%$ CI: 0.58-1.15; $p=0.242)$. In terms of RCI ( $50 \%$ increase) the onlinebased treatment was associated with 1 case of deterioration at the posttreatment assessment for every 41.43 (95\% 
CI: $22.58-250.08)$ [104.35 (95\% CI: $\left.33.35-10^{6}\right)$ ] participants who received treatment. The NNTHs for FU1 and FU2 were 96.84 (95\% CI: 31.35-106) [129.54 (95\% CI: $\left.36.99-10^{6}\right)$ and 22.44 (95\% CI: $\left.12.53-107.20\right)$ [38.82 (95\% CI: $\left.17.06-10^{6}\right)$ ], respectively.

\section{Effects on Depression Onset}

Three of the 7 studies with $n=1,583$ participants $(72.4 \%$ of the whole sample) provided data on time to onset of $\operatorname{MDD}[20,25,73]$. In total, $18.7 \%$ of the participants in the IG and $25.8 \%$ in the CG experienced the onset of MDD during the study period. Online supplement 7 shows the Kaplan-Meier survival curves for the IG and CG generated for the 12-month study period. The Kaplan-Meier estimates of the cumulative incidence of MDD were $26 \%$ (95\% CI: $22-30 \%$ ) for the IG and 34\% (95\% CI: 30-37\%) for the CG. The log-rank test revealed a statistically significant difference between incidence rates over time ( $p=$ 0.004). The mean time to onset of MDD within the 12-month trial period was 33 weeks in the IG and 32 weeks in the CG. Cox regression, which controlled for initial DSS, revealed a hazard ratio of 0.72 (95\% CI: 0.58-0.89), which means that the risk of developing an MDD within 12 months is reduced by $28 \%$ in the IG compared to the CG when controlled for initial DSS. The estimated hazard ratio for DSS at baseline was 1.09 (95\% CI: 1.06-1.11). There was no evidence for non-constant hazard ratios (global test of non-proportionality, $p=0.568$; treatment condition, $p=0.501$; DSS, $p=0.689)$.

\section{Moderators of Intervention Effects on DSS}

Results of separate multilevel regression analyses, each examining interactions of moderator $x$ intervention effects, are displayed in Table 4. Results indicated initial DSS $(p=0.008)$ as well as age ( $p=0.045)$ to be significant moderators of short-term treatment effects, with high initial DSS and older age associated with greater intervention effects (online supplements 8,9). Afterwards, this analysis was repeated with both identified moderators and their interaction effects in one model to determine whether they are independent of one another. Results remained the same (online suppl. supplement 10).

Neither any of the sociodemographic characteristics (sex, relationship status, employment, education) nor any other clinical characteristics (anxiety symptoms, comorbid mental health disorder, previous psychotherapy for depression, use of antidepressants, chronic medical conditions) or the length of treatment on study level were associated with differential treatment effects. Guided format hardly reached statistical significance $(p=0.057)$.

IPD-MA on Internet-Based Interventions in Adults with Subthreshold Depression

\section{Discussion}

\section{Main Findings}

We found that Internet-based interventions resulted in lower DSS, greater treatment response and close to symptom-free status at posttreatment assessment, FU1 and FU2, and a reduced risk of depression onset within 12 months compared with control conditions. A reliable symptom deterioration was found for the CG at the posttreatment time point and at FU2 but no significant differences between groups were found for symptom deterioration in terms of $50 \%$ symptom increase. Results of the moderator analyses indicated higher DSS and older age to be associated with statistically significantly greater short-term treatment effects. Guided interventions showed better effects compared to unguided interventions, but this effect barely reached statistical significance.

\section{Comparison to Previous Research}

The between-group effect sizes of $g=0.39,0.30$ and 0.27 found in our IPD-MA for reducing DSS at posttreatment assessment, FU1 and FU2, respectively, are somewhat higher than those reported in recent meta-analyses on the effects of preventive Internet-based interventions for the treatment of $\mathrm{sD}(\mathrm{d}=0.25$ at posttreatment [15], $\mathrm{d}$ $=0.28$ at posttreatment $[16] ; \mathrm{d}=0.35,0.22,0.14$ at posttreatment, FU1 and FU2, respectively [17]). The greater effect sizes found in our IPD-MA could be explained by a higher symptom severity at baseline as we only included indicated prevention studies that permit a greater potential for improvement, while Zhou at al. [16] also included universal prevention studies. The meta-analysis by Deady et al. [15] further included a study that evaluated a transdiagnostic trait-focused Internet-based intervention aimed at reducing symptoms of common mental disorders in university students [76], which is not directly comparable to cognitive-behavioural approaches used in other prevention studies. Furthermore, Sander et al. [17] included different interventions targeting a variety of indications like eating disorders or posttraumatic stress disorders with depression being only a secondary outcome in some cases, and there were also universal prevention studies included.

Previous meta-analyses based on comparisons of means could provide only little information regarding response and close to symptom-free status on an individual level. However, a recent IPD-MA on Internet-based interventions in patients with MDD found the IG to obtain significantly higher response and remission rates compared to controls after treatment (5-12 weeks) [47]. Ac- 
cordingly, we have been able to show short-term effects on the intervention group in the period of up to 3-6 months after treatment in this IPD-MA as well. The positive effect persists up to 12 months.

There were no significant differences between the groups in deterioration rates in terms of $50 \%$ symptom increase but the IG was superior with fewer cases of reliable deterioration at the posttreatment assessment and FU2 compared to the CG. We found the risk for deterioration to be decreased by $35 \%$ in the IG compared to the CG. The latter finding points in the same direction like the results from a recent IPD analysis on MDD, which found the risk for a reliable deterioration from baseline to posttreatment time point to be significantly lower in the intervention versus control condition (relative risk = 0.47, 95\% CI: 0.29-0.75) [77].

We found a risk reduction of $28 \%$ to develop an MDD within a year. This is similar to the results in a meta-analysis on preventive interventions for depression including 17 studies for indicated prevention, which found a mean risk reduction of $26 \%$ [78].

With regard to moderators, initial symptom severity and age were identified as moderators on DSS at posttreatment assessment. Guided format just missed the significance level.

The higher the initial DSS, the lower the depressive symptomatology at posttreatment assessment in the IG compared to the CG. Previous research on depression prevention interventions showed inconsistent results with one trial that found low initial DSS leads to a better outcome [79], while two other trials found the opposite effect $[80,81]$ and several trials that did not find any moderating effect at all (e.g., $[82,83])$. Evidence on the impact of initial DSS in the field of MDD is conflicting as well (e.g., $[47,84,85])$. Given limited health care resources, future studies should identify the level of depression with an ideal cost-benefit ratio. Furthermore, higher age was associated with greater intervention effects on DSS at posttreatment assessment as well. This was also found in a recent meta-analysis based on individual participant data (IPD-MA) on Internet-based treatment of MDD [47] but in the field of sD several single trials led to mixed results: Vázquez et al. [82] for example showed that younger nurses benefited more from the Internet-based cognitive-behavioural therapy (iCBT) programme than older ones while age was not shown to be a significant moderator for treatment success when comparing iCBT versus routine care in a study by Button et al. [61]. There was only a trend for guided format to be associated with greater effects compared to purely self-guided interven- tions. There are no other meta-analyses or reviews yet investigating guidance as a moderator in Internet interventions for $\mathrm{sD}$, but another single trial did not find semistandardized guidance in iCBT for mild to moderate depression to be more effective than fully standardized feedback on DSS but leading to higher attrition rates [86]. In the field of MDD, numerous meta-analyses of recent years showed a superiority of the interventions with guidance compared to no guidance $[64,87,88]$. No other moderators were identified. While Richards and Richardson [64] found the pooled effect size for studies evaluating Internet-based interventions for depression which used less than 8 sessions to be considerably higher than studies which used 8 or more sessions in participants with MDD, this could not be confirmed in our analysis for people with sD.

\section{Clinical Implications and Recommendations for}

Future Research

Results from this IPD-MA have important implications:

First, so far there are no obvious reasons to exclude specific subgroups of individuals, as intervention outcome has been shown to be independent of gender, relationship status, employment status, comorbid mental health disorders, chronic medical conditions as well as previous depression treatment or use of antidepressants. Moreover, there seems to be a lower risk for deterioration and for depression onset when taking part in Internetbased interventions compared to treatment as usual. However, more research is needed to evaluate the potential of iCBT in specific subgroups (e.g., younger age groups, low initial symptom severity) to determine whether or not they benefit from this kind of interventions, not only related to the prevention of depression onset but also to other outcomes, such as quality of life. In particular, more research is necessary to assess the point at which depressive symptoms at the lower end of the severity spectrum become sufficiently persistent to warrant preventive interventions. It might be beneficial to tailor Internet-based interventions to the specific needs of these subgroups to achieve greater treatment effects. Since gender was not a significant moderator, more effort should be put into also reaching men who are experiencing depressive symptoms, who are usually underrepresented in preventive interventions, as they are likely to benefit equally to women. For example, recruitment strategies as well as male-specific adaptations to interventions might be needed to improve intervention uptake. Future studies should identify preferences for and 
barriers to participation in preventive psychological interventions in general but especially among men [10]. Some evidence suggests that pre-intervention motivational interviewing might increase help-seeking among men [89].

Second, the role of guidance has not yet been sufficiently explored. While a guided digital intervention format seems to lead to an additional benefit for individuals with MDD, the availability of individualized feedback for people suffering from $\mathrm{sD}$ probably has less impact on the effectiveness of the Internet-based intervention. Furthermore, it has been argued that effect sizes for unguided Internet-based interventions found in RCTs might be overestimated for what can be expected in routine care, as the structure in clinical trials (e.g., clinical interviews and structured outcome assessment) might be an adherence-fostering element [90], which is assumed an important underlying mechanism of the treatment effect [91]. Moreover, individuals might be less motivated to participate in interventions in which no regular content feedback from a psychologist is offered, which would lead to a lower reach and thus lower overall effects in the target population. This assumption was supported by a web-based survey study on public attitudes toward guided Internet-based therapies [92]. However, the potential on a population level might still be high, as such interventions can be distributed to more participants within a given health care budget. A stepped care model could be kind of a compromise when affected individuals usually start using an unguided intervention in a first step and receive guidance in the case of non-response (second step).

\section{Strengths and Limitations}

A strength of this IPD-MA is the sufficient statistical power to provide information on the long-term effects of psychological Internet-based interventions, which had been largely unclear and inconsistent so far. In addition, conclusions regarding response, remission, symptom deterioration and depression onset could also be drawn from the study data. Using an IPD-MA approach allowed us to analyse moderating effects and to test whether the efficacy depends on individual participant criteria. This leads to crucial insights about best possible treatments for specific subgroups. Furthermore, the methodological quality of the included studies was very high so that we can assume that the results are robust.

However, the study also has some limitations. First, two thirds of participants were at least highly educated ( $>13$ years of education). Hence, the generalizability of

IPD-MA on Internet-Based Interventions in Adults with Subthreshold Depression our results might be limited to participants with high educational levels. However, education was not a significant moderator of treatment effect. Thus, although low educated people are usually underrepresented in clinical trials, they might benefit from iCBT as well [47]. In addition, more research is needed to evaluate treatment effects in people with low socio-economic status and having low Internet affinity. Second, DSS was only assessed with self-report instruments. Depending on the symptom severity of an individual, clinician ratings or self-report might be more suitable. Therefore, it seems best to include both, clinician rating and self-report in clinical research [93]. Third, the estimate of the slope variance at FU2 is very large and so are the standard errors for the fixed effects in that model. This is likely an effect of the extreme rate of missing data for end points at FU2. Fourth, we were unable to consider further moderators in our analysis, because many relevant variables associated with differential treatment response or depression incidence in the literature, such as lifetime history of depression, childhood adversity, personal characteristics, quality of life or mastery, had not been included in many of the published studies, leading to high rates of missing data and cases in which study level characteristics were completely confounded with missingness. More studies are ultimately needed to provide insight into the effects of additional moderators. Special attention should be paid to a more specific investigation of the influence of pharmacotherapy in patients with subclinical depression and its interaction with Internet-based treatments [26]. Finally, because this is an emerging field there are still contradictory results in the literature on effect moderators. Therefore, even a meta-analysis with high statistical power and sophisticated and carefully performed statistical methods is unlikely to provide a concluding answer at this point of time $[94,95]$. With a higher number of primary studies and therefore even higher statistical power, more knowledge about moderating effects might be gained in the nearby future.

\section{Conclusions}

The findings of this IPD-MA provide evidence for Internet-based interventions to be a suitable low-threshold intervention to treat individuals with subclinical depressive symptoms and a wide range of participant characteristics and, moreover, to reduce the incidence of MDD. Effects were especially pronounced for older participants when initial DSS was already substantial. 


\section{Statement of Ethics}

The authors assert that all procedures contributing to this work comply with the ethical standards of the relevant national and institutional committees on human experimentation and with the Helsinki Declaration of 1975, as revised in 2008.

\section{Disclosure Statement}

D.D.E. and D.L. reported holding minor shares of the Institute for OnlineHealth Training (GET.ON), which aims to transfer scientific knowledge related to the present research into routine mental health care. This institute licenses some interventions under study $[20,21,71,96]$ from the Leuphana University, Lueneburg, to provide the intervention within routine preventive services of health insurance companies in Germany.

\section{Funding Sources}

There was no specific funding for this work.

\section{Author Contributions}

D.D.E. conceptualized and designed the study, P.C. contacted the primary authors, J.R. and C.B. were responsible for building the database with support from K.W. and M.D. J.Z. and M.H. were responsible for the data analyses, S.G. and J.Z. for the imputation procedure. J.R. drafted the manuscript, supervised by D.E. and C.B. All authors critically revised the paper, read and approved the final version.

\section{References}

1 Cuijpers P, de Graaf R, van Dorsselaer S. Minor depression: risk profiles, functional disability, health care use and risk of developing major depression. J Affect Disord. 2004 Apr; 79(1-3):71-9.

2 Rucci P, Gherardi S, Tansella M, Piccinelli M, Berardi D, Bisoffi G, et al. Subthreshold psychiatric disorders in primary care: prevalence and associated characteristics. J Affect Disord. 2003 Sep;76(1-3):171-81.

3 Lee YY, Stockings EA, Harris MG, Doi SA, Page IS, Davidson SK, et al. The risk of developing major depression among individuals with subthreshold depression: a systematic review and meta-analysis of longitudinal cohort studies. Psychol Med. 2019 Jan;49(1): 92-102.

4 Cuijpers P, Smit F. Subthreshold depression as a risk indicator for major depressive disorder: a systematic review of prospective studies. Acta Psychiatr Scand. 2004 May; 109(5): $325-31$.

5 Cuijpers P, Vogelzangs N, Twisk J, Kleiboer A, Li J, Penninx BW. Differential mortality rates in major and subthreshold depression: meta-analysis of studies that measured both. Br J Psychiatry. 2013 Jan;202(1):22-7.

6 Goldney RD, Fisher LJ, Dal Grande E, Taylor AW. Subsyndromal depression: prevalence, use of health services and quality of life in an Australian population. Soc Psychiatry Psychiatr Epidemiol. 2004 Apr;39(4):293-8.

7 Cuijpers P, Smit F, Oostenbrink J, de Graaf R, Ten Have M, Beekman A. Economic costs of minor depression: a population-based study. Acta Psychiatr Scand. 2007 Mar;115(3):22936.

8 Cuijpers P, Koole SL, van Dijke A, Roca M, Li J, Reynolds CF 3rd. Psychotherapy for subclinical depression: meta-analysis. Br J Psychiatry. 2014 Oct;205(4):268-74.

9 Cuijpers P, van Straten A, Warmerdam L, van Rooy MJ. Recruiting participants for inter- ventions to prevent the onset of depressive disorders: possible ways to increase participation rates. BMC Health Serv Res. 2010 Jun; 10(1): 181

10 Ebert DD, Cuijpers P, Muñoz RF, Baumeister $H$. Prevention of mental health disorders using Internet- and mobile-based interventions: a narrative review and recommendations for future research. Front Psychiatry. 2017 Aug; 8:116.

11 Andrade LH, Alonso J, Mneimneh Z, Wells JE, Al-Hamzawi A, Borges G, et al. Barriers to mental health treatment: results from the WHO World Mental Health surveys. Psychol Med. 2014 Apr;44(6):1303-17.

12 Wright JH, Owen JJ, Richards D, Eells TD, Richardson T, Brown GK, et al. Computerassisted cognitive-behavior therapy for depression: A systematic review and meta-analysis. J Clin Psychiatry. 2019 Mar;80(2): $18 \mathrm{r} 12188$

13 Königbauer J, Letsch J, Doebler P, Ebert DD. Internet- and mobile-based depression interventions for people with diagnosed depression: a systematic review and metaanalysis. J Affect Disord. 2017 Dec;223:2840.

14 NICE - National Institute for Health and Care Excellence. Depression in adults: recognition and management. Clinical guideline [CG90] [Internet]. 2009. Available from: https://www.nice.org.uk/guidance/cg90/ chapter/1-Guidance\#step-1-recognition-assessment-and-initial-management

15 Deady M, Choi I, Calvo RA, Glozier N, Christensen $\mathrm{H}$, Harvey SB. eHealth interventions for the prevention of depression and anxiety in the general population: a systematic review and meta-analysis. BMC Psychiatry. 2017 Aug; 17(1):310.

16 Zhou T, Li X, Pei Y, Gao J, Kong J. Internetbased cognitive behavioural therapy for subthreshold depression: a systematic review and meta-analysis. BMC Psychiatry. 2016 Oct; 16(1):356.

17 Sander L, Rausch L, Baumeister H. Effectiveness of Internet-based interventions for the prevention of mental disorders: a systematic review and meta-analysis. JMIR Ment Health; 2016 Aug;3(3):e38.

18 Jacobson NS, Truax P. Clinical significance: a statistical approach to defining meaningful change in psychotherapy research. J Consult Clin Psychol. 1991 Feb;59(1):12-9.

19 Spek V, Cuijpers P, Nyklícek I, Smits N, Riper $\mathrm{H}$, Keyzer J, et al. One-year follow-up results of a randomized controlled clinical trial on internet-based cognitive behavioural therapy for subthreshold depression in people over 50 years. Psychol Med. 2008 May;38(5):635-9.

20 Buntrock C, Ebert D, Lehr D, Riper H, Smit F, Cuijpers $\mathrm{P}$, et al. Effectiveness of a web-based cognitive behavioural intervention for subthreshold depression: pragmatic randomised controlled trial. Psychother Psychosom. 2015; 84(6):348-58.

21 Buntrock C, Ebert DD, Lehr D, Smit F, Riper $\mathrm{H}$, Berking $\mathrm{M}$, et al. Effect of a web-based guided self-help intervention for prevention of major depression in adults with subthreshold depression: a randomized clinical trial. JAMA. 2016 May;315(17):1854-63.

22 Imamura K, Kawakami N, Tsuno K, Tsuchiya M, Shimada K, Namba K. Effects of webbased stress and depression literacy intervention on improving symptoms and knowledge of depression among workers: a randomized controlled trial. J Affect Disord. 2016 Oct;203: $30-7$.

23 Phillips R, Schneider J, Molosankwe I, Leese M, Foroushani PS, Grime P, et al. Randomized controlled trial of computerized cognitive behavioural therapy for depressive symptoms: effectiveness and costs of a workplace intervention. Psychol Med. 2014 Mar;44(4): 741-52. 
24 Proudfoot J, Clarke J, Birch MR, Whitton AE, Parker G, Manicavasagar V, et al. Impact of a mobile phone and web program on symptom and functional outcomes for people with mild-to-moderate depression, anxiety and stress: a randomised controlled trial. BMC Psychiatry. 2013 Nov;13(1):312.

25 Imamura K, Kawakami N, Furukawa TA, Matsuyama Y, Shimazu A, Umanodan R, et al. Does Internet-based cognitive behavioral therapy (iCBT) prevent major depressive episode for workers? A 12-month follow-up of a randomized controlled trial. Psychol Med. 2015 Jul;45(9):1907-17.

26 Guidi J, Brakemeier EL, Bockting CL, Cosci F, Cuijpers P, Jarrett RB, et al. Methodological Recommendations for Trials of Psychological Interventions. Psychother Psychosom. 2018; 87(5):276-84.

27 Brookes ST, Whitely E, Egger M, Smith GD, Mulheran PA, Peters TJ. Subgroup analyses in randomized trials: risks of subgroup-specific analyses; power and sample size for the interaction test. J Clin Epidemiol. 2004 Mar;57(3): 229-36.

28 Kraemer HC, Wilson GT, Fairburn CG, Agras WS. Mediators and moderators of treatment effects in randomized clinical trials. Arch Gen Psychiatry. 2002 Oct;59(10):87783.

29 Cooper H, Patall EA. The relative benefits of meta-analysis conducted with individual participant data versus aggregated data. Psychol Methods. 2009 Jun;14(2):165-76.

30 Stewart LA, Clarke M, Rovers M, Riley RD, Simmonds M, Stewart G, et al; PRISMAIPD Development Group. Preferred reporting items for a systematic review and metaanalysis of individual participant data: the PRISMA-IPD statement. JAMA. 2015 Apr; 313(16):1657-65.

31 Tierney JF, Vale C, Riley R, Smith CT, Stewart $\mathrm{L}$, Clarke M, et al. Individual participant data (IPD) metaanalyses of randomised controlled trials: guidance on their use. PLoS Med. 2015 Jul;12(7):e1001855.

32 Ebert DD, Buntrock C, Reins JA, Zimmermann J, Cuijpers P. Efficacy and moderators of psychological interventions in treating subclinical symptoms of depression and preventing major depressive disorder onsets: protocol for an individual patient data meta-analysis of randomised controlled trials. BMJ Open. 2018 Mar;8(3):e018582.

33 Cuijpers P, Karyotaki E, Ciharova M. A metaanalytic database of randomised trials on psychotherapies for depression [Internet]. OSF; 2019. Available from: https://doi.org/ 10.17605/OSF.IO/825C6.

34 Kessler RC, van Loo HM, Wardenaar KJ, Bossarte RM, Brenner LA, Cai T, et al. Testing a machine-learning algorithm to predict the persistence and severity of major depressive disorder from baseline self-reports. Mol Psychiatry. 2016 Oct;21(10):1366-71.

35 Kessler RC, van Loo HM, Wardenaar KJ, Bossarte RM, Brenner LA, Ebert DD, et al. Us- ing patient self-reports to study heterogeneity of treatment effects in major depressive disorder. Epidemiol Psychiatr Sci. 2017 Feb;26(1): 22-36.

36 Cuijpers P, Andersson G, Donker T, van Straten A. Psychological treatment of depression: results of a series of meta-analyses. Nord J Psychiatry. 2011 Dec;65(6):354-64.

37 Higgins JPT, Savović J, Page MJ, Sterne JA. Revised Cochrane risk-of-bias tool for randomized trials (RoB 2) [Internet]. Full Guide Doc. 2019. Available from: riskofbias.info

38 van Buuren S, Groothuis-Oudshoorn K. mice: multivariate imputation by chained equations in R. J Stat Softw. 2011;45(3). DOI: 10.18637/jss.v045.i03.

39 Quartagno M, Carpenter JR. Multiple imputation for IPD meta-analysis: allowing for heterogeneity and studies with missing covariates. Stat Med. 2016 Jul;35(17):2938-54.

40 Lüdtke O, Robitzsch A, Grund S. Multiple imputation of missing data in multilevel designs: A comparison of different strategies. Psychol Methods. 2017 Mar;22(1):141-65.

41 Jolani S, Debray TP, Koffijberg H, van Buuren S, Moons KG. Imputation of systematically missing predictors in an individual participant data meta-analysis: a generalized approach using MICE. Stat Med. 2015 May; 34(11):1841-63.

42 Resche-Rigon M, White IR. Multiple imputation by chained equations for systematically and sporadically missing multilevel data. Stat Methods Med Res. 2018 Jun;27(6):1634-49.

43 Drechsler J. Multiple imputation of multilevel missing data - rigor versus simplicity. J Educ Behav Stat. 2015;40(1):69-95.

44 McCoach DB, Rifenbark GG, Newton SD, Li X, Kooken J, Yomtov D, et al. Does the package matter? A comparison of five common multilevel modeling software packages. J Educ Behav Stat. 2018;43(5):594-627.

45 Beck AT, Steer RA, Brown GK. Manual for the Beck Depression Inventory-II. San Antonio (TX): Psychological Corporation; 1996.

46 Wang YP, Gorenstein C. Assessment of depression in medical patients: a systematic review of the utility of the Beck Depression Inventory-II. Clinics (São Paulo). 2013 Sep; 68(9):1274-87.

47 Karyotaki E, Ebert DD, Donkin L, Riper H, Twisk J, Burger S, et al. Do guided internetbased interventions result in clinically relevant changes for patients with depression? An individual participant data meta-analysis. Clin Psychol Rev. 2018 Jul;63:80-92.

48 Beck AT, Steer A, Brown GK. BDI-II: Beck Depression Inventory manual. 2nd ed. San Antonio: Psychological Corporation; 1996.

49 Radloff LS. The CES-D scale: a self-report depression scale for research in the general population. Appl Psychol Meas. 1977;1(3):385-401.

50 Wahl I, Löwe B, Bjorner JB, Fischer F, Langs $\mathrm{G}$, Voderholzer U, et al. Standardization of depression measurement: a common metric was developed for 11 self-report depression measures. J Clin Epidemiol. 2014 Jan;67(1):73-86.
51 Miletic V, Lukovic JA, Ratkovic N, Aleksic D, Grgurevic A. Demographic risk factors for suicide and depression among Serbian medical school students. Soc Psychiatry Psychiatr Epidemiol. 2015 Apr;50(4):633-8.

52 Sajjadi H, Mohaqeqi Kamal SH, Rafiey H, Vameghi M, Forouzan AS, Rezaei M. A systematic review of the prevalence and risk factors of depression among iranian adolescents. Glob J Health Sci. 2013 Jan;5(3):16-27.

53 Yanzón de la Torre A, Oliva N, Echevarrieta PL, Pérez BG, Caporusso GB, Titaro AJ, et al. Major depression in hospitalized Argentine general medical patients: prevalence and risk factors. J Affect Disord. 2016 Jun;197:36-42.

54 Kounali D, Zammit S, Wiles N, Sullivan S, Cannon M, Stochl J, et al. Common versus psychopathology-specific risk factors for psychotic experiences and depression during adolescence. Psychol Med. 2014 Sep;44(12): 2557-66.

55 Hölzel L, Härter M, Reese C, Kriston L. Risk factors for chronic depression-a systematic review. J Affect Disord. 2011 Mar;129(1-3): $1-13$.

56 Heslin M, Desai R, Lappin JM, Donoghue K, Lomas B, Reininghaus U, et al. Biological and psychosocial risk factors for psychotic major depression. Soc Psychiatry Psychiatr Epidemiol. 2016 Feb;51(2):233-45.

57 Warmerdam L, Van Straten A, Twisk J, Cuijpers P. Predicting outcome of Internet-based treatment for depressive symptoms. Psychother Res. 2013;23(5):559-67.

58 Daoud N, O’Brien K, O'Campo P, Harney S, Harney E, Bebee K, et al. Postpartum depression prevalence and risk factors among Indigenous, non-Indigenous and immigrant women in Canada. Can J Public Health. 2019 Aug; 110(4):440-52.

59 Støen Grotmol K, Gude T, Moum T, Vaglum P, Tyssen R. Risk factors at medical school for later severe depression: a 15-year longitudinal, nationwide study (NORDOC). J Affect Disord. 2013 Mar;146(1):106-11.

60 Johansson R, Sjöberg E, Sjögren M, Johnsson E, Carlbring P, Andersson T, et al. Tailored vs. standardized internet-based cognitive behavior therapy for depression and comorbid symptoms: a randomized controlled trial. PLoS One. 2012;7(5):e36905.

61 Button KS, Wiles NJ, Lewis G, Peters TJ, Kessler D. Factors associated with differential response to online cognitive behavioural therapy. Soc Psychiatry Psychiatr Epidemiol. 2012 May;47(5):827-33.

62 de Graaf LE, Hollon SD, Huibers MJ. Predicting outcome in computerized cognitive behavioral therapy for depression in primary care: A randomized trial. J Consult Clin Psychol. 2010 Apr;78(2):184-9.

63 Bromberger JT, Schott L, Kravitz HM, Joffe $\mathrm{H}$. Risk factors for major depression during midlife among a community sample of women with and without prior major depression: are they the same or different? Psychol Med. 2015 Jun;45(8):1653-64. 
64 Richards D, Richardson T. Computer-based psychological treatments for depression: a systematic review and meta-analysis. Clin Psychol Rev. 2012 Jun;32(4):329-42.

65 Kontopantelis E. A comparison of one-stage vs two-stage individual patient data metaanalysis methods: A simulation study. Res Synth Methods. 2018 Sep;9(3):417-30.

66 Gladitz J. Rubin, Donald B. Multiple imputation for nonresponse in surveys. John Wiley \& Sons, Chichester - New York - Brisbane Toronto - Singapore 1987, xxx, 258 S., 6 Abb., $£$ 30.25, ISSN 0271-6232. Biometrical J. 1989; 31(1):131-2.

67 Douglas Bates MM, Bates D, Mächler M, Bolker B. Fitting linear mixed-effects models using lme4. J Stat Softw. 2015 Oct;67(1):1-48.

68 Grund S, Robitzsch A, Luedtke O. mitml: tools for multiple imputation in multilevel modeling [Internet]. Version 03-7. 2019 [cited 2019 Jul 1]. Available from: https://cran.rproject.org/package $=$ mitml

69 Laupacis A, Sackett DL, Roberts RS. An assessment of clinically useful measures of the consequences of treatment. N Engl J Med. 1988 Jun;318(26):1728-33.

70 Schoenfeld D. Partial residuals for the proportional hazards regression model. Biometrika. 1982;69(1):239-41.

71 Nobis S, Lehr D, Ebert DD, Baumeister H, Snoek F, Riper H, et al. Efficacy of a webbased intervention with mobile phone support in treating depressive symptoms in adults with type 1 and type 2 diabetes: a randomized controlled trial. Diabetes Care. 2015 May;38(5):776-83.

72 van Bastelaar KM, Pouwer F, Cuijpers P, Riper H, Snoek FJ. Web-based depression treatment for type 1 and type 2 diabetic patients: a randomized, controlled trial. Diabetes Care. $2011 \mathrm{Feb} ; 34(2): 320-5$.

73 Klein JP, Berger T, Schröder J, Späth C, Meyer B, Caspar F, et al. Effects of a psychological internet intervention in the treatment of mild to moderate depressive symptoms: results of the evident study, a randomized controlled trial. Psychother Psychosom. 2016;85(4): $218-28$.

74 Imamura K, Kawakami N, Furukawa TA, Matsuyama Y, Shimazu A, Umanodan R, et al. Effects of an Internet-based cognitive behavioral therapy (iCBT) program in Manga format on improving subthreshold depressive symptoms among healthy workers: a randomized controlled trial. PLoS One. 2014 May;9(5):e97167.
75 Spek V, Nyklícek I, Smits N, Cuijpers P, Riper $\mathrm{H}$, Keyzer J, et al. Internet-based cognitive behavioural therapy for subthreshold depression in people over 50 years old: a randomized controlled clinical trial. Psychol Med. 2007 Dec;37(12):1797-806.

76 Musiat P, Conrod P, Treasure J, Tylee A, Williams C, Schmidt U. Targeted prevention of common mental health disorders in university students: randomised controlled trial of a transdiagnostic trait-focused web-based intervention. PLoS One. 2014 Apr;9(4):e93621.

77 Ebert DD, Donkin L, Andersson G, Andrews G, Berger T, Carlbring P, et al. Does Internetbased guided-self-help for depression cause harm? An individual participant data metaanalysis on deterioration rates and its moderators in randomized controlled trials. Psychol Med. 2016 Oct;46(13):2679-93.

78 van Zoonen K, Buntrock C, Ebert DD, Smit F, Reynolds CF 3rd, Beekman AT, et al. Preventing the onset of major depressive disorder: a meta-analytic review of psychological interventions. Int J Epidemiol. 2014 Apr;43(2): $318-29$.

79 Allart-van Dam E, Hosman CM, Hoogduin CA, Schaap CP. Prevention of depression in subclinically depressed adults: follow-up effects on the 'Coping with Depression' course. J Affect Disord. 2007 Jan;97(1-3):219-28.

80 Lara MA, Navarro C, Navarrete L. Outcome results of a psycho-educational intervention in pregnancy to prevent $\mathrm{PPD}$ : a randomized control trial. J Affect Disord. 2010 Apr;122(12):109-17.

81 Barrera AZ, Wickham RE, Muñoz RF. Online prevention of postpartum depression for Spanish- and English-speaking pregnant women: A pilot randomized controlled trial. Internet Interv. 2015 Sep;2(3):257-65.

82 Vázquez FL, Torres Á, Blanco V, Otero P, Díaz O, Ferraces MJ. Long-term Follow-up of a Randomized Clinical Trial Assessing the Efficacy of a Brief Cognitive-Behavioral Depression Prevention Intervention for Caregivers with Elevated Depressive Symptoms. Am J Geriatr Psychiatry. 2016 Jun;24(6):421-32.

83 Otero P, Smit F, Cuijpers P, DeRubeis RJ, Torres Á, Vázquez FL. Differential response to depression prevention among a sample of informal caregivers: moderator analysis of longer-term follow-up trial data. Psychiatry Res. 2015 Dec;230(2):271-8.

84 Bower P, Kontopantelis E, Sutton A, Kendrick T, Richards DA, Gilbody S, et al. Influence of initial severity of depression on effectiveness of low intensity interventions: metaanalysis of individual patient data. BMJ. 2013 Feb;346:f540.
85 Karyotaki E, Riper H, Twisk J, Hoogendoorn A, Kleiboer A, Mira A, et al. Efficacy of Selfguided Internet-Based Cognitive Behavioral Therapy in the Treatment of Depressive Symptoms: A Meta-analysis of Individual Participant Data. JAMA Psychiatry. 2017 Apr;74(4):351-9.

86 Zagorscak P, Heinrich M, Sommer D, Wagner B, Knaevelsrud C. Benefits of Individualized Feedback in Internet-Based Interventions for Depression: A Randomized Controlled Trial. Psychother Psychosom. 2018; 87(1):32-45.

87 Baumeister H, Reichler L, Munzinger M, Lin $\mathrm{J}$. The impact of guidance on Internet-based mental health interventions - A systematic review. Internet Interv. 2014;1(4):205-15.

88 Cowpertwait L, Clarke D. Effectiveness of web-based psychological interventions for depression: a meta-analysis. Int J Ment Health Addict. 2013;11(2):247-68.

89 Syzdek MR, Addis ME, Green JD, Whorley MS, Berger JL. A pilot trial of gender-based motivational interviewing for help-seeking and internalizing symptoms in men. Psychol Men Masc. 2014 Jan;15(1):90-4.

90 Ebert DD, Baumeister H. Internet-based selfhelp interventions for depression in routine care. JAMA Psychiatry. 2017 Aug;74(8):8523.

91 Ebert DD, Van Daele T, Nordgreen T, Karekla $\mathrm{M}$, Compare A, Zarbo C, et al. Internet- and mobile-based psychological interventions: applications, efficacy, and potential for improving mental health: a report of the EFPA E-Health Taskforce. Eur Psychol. 2018;23(2): 167-87.

92 Apolinário-Hagen J, Harrer M, Kählke F, Fritsche L, Salewski C, Ebert DD. Public attitudes toward guided internet-based therapies: web-based survey study. JMIR Ment Health. 2018 May;5(2):e10735.

93 Cuijpers P, Li J, Hofmann SG, Andersson G. Self-reported versus clinician-rated symptoms of depression as outcome measures in psychotherapy research on depression: a meta-analysis. Clin Psychol Rev. 2010 Aug;30(6): 768-78.

94 Concato J, Horwitz RI. Limited Usefulness of Meta-Analysis for Informing Patient Care. Psychother Psychosom. 2019;88(5):257-62.

95 de Vrieze J. The metawars. Science. 2018 Sep; 361(6408):1184-8.

96 Ebert DD, Buntrock C, Lehr D, Smit F, Riper $\mathrm{H}$, Baumeister H, et al. Effectiveness of Weband Mobile-Based Treatment of Subthreshold Depression With Adherence-Focused Guidance: A Single-Blind Randomized Controlled Trial. Behav Ther. 2018 Jan;49(1):71-83. 